\title{
G estion par les feux des pâturages naturels et productivité des bovins sur les prairies des basses plaines orientales de Colombie
}

\author{
G. Rippstein ${ }^{1}$ G. Allard ${ }^{2}$ J. Corbin ${ }^{3}$
}

\begin{abstract}
Mots-clés
Bovin - Système de pâturage Conduite des herbages - Taux de charge - Pâturage en rotation Repousse - Valeur nutritive - Plaine Colombie.
\end{abstract}

\begin{abstract}
Résumé
La productivité et la valeur alimentaire des différents types de pâturages naturels des basses plaines orientales de Colombie (Llanos), gérées par des feux annuels de saison sèche, ont été généralement faibles : les biomasses aériennes annuelles ont été de l'ordre de 2 à 3,5 tonnes de matière sèche par hectare et les teneurs en matières azotées totales des fourrages ont été comprises entre 5 et 11 p. 100 de la matière sèche suivant les saisons et le stade phénologique. $D$ ans les conditions traditionnelles d'exploitation (pâture continue, pas de feu ou des feux en début de saison sèche, chargement de 8-10 ha par animal), les gains de poids des bovins ont été faibles en saison des pluies (gains quotidiens moyens entre 150 à $300 \mathrm{~g}$ ) et ils ont perdu généralement du poids en saison sèche (de 150 à $250 \mathrm{~g}$ par jour). D es expérimentations en station ont montré une différence significative de productivité des animaux selon la composition botanique, ou mieux, la valeur pastorale (indice synthétique de valeur du pâturage intégrant productivité, valeur nutritive et appétence des espèces) des pâturages naturels suivant qu'ils étaient sur des sols à texture sablo-argileuse ou argilo-limoneuse. Les feux annuels de saison sèche ont permis la production de repousses vertes mais, répétés d'année en année et associés à la libre pâture en continu, ils ont dégradé la flore. Les auteurs ont testé, sur un ensemble de parcelles en rotation, deux systèmes séquentiels de feux permettant de les espacer de 16 mois et de mettre à la disposition du bétail des repousses protégées de la pâture toutes les quatre semaines. Malgré un doublement de la charge moyenne annuelle (4 ha par tête), les gains de poids individuels de bovins en croissance ont été significativement améliorés et ce résultat a été obtenu sans dégradation de la végétation.
\end{abstract}

\section{INTRO DUCTION}

Le feu, dans les savanes humides du monde entier, « ne doit pas être considéré comme un facteur de transformation (ou de dégradation), mais comme un facteur de conservation (et de gestion) des savanes ; il est nécessaire pour maintenir le cortège floristique de la savane, en particulier de la strate graminéenne, indispensable à l'élevage » $(5,9)$.

1. Cirad-emvt/Ciat, TA 30/B, Campus international de Baillarguet, 34398 Montpellier Cedex 5, France

Tél. : +33 (0) 467593812 ; fax : +33 (0) 467593799 ;

e-mail : georges.rippstein@cirad.fr

2. Université Paris XII, France

3. Istom, Cergy, France
Dans la plupart des vastes prairies naturelles des basses plaines, les Llanos d'Amérique du Sud, en particulier dans celles de l'est de la Colombie et du sud-ouest du Venezuela, les feux de début de saison sèche sont indispensables pour la gestion des pâturages naturels (15) :

- ils permettent d'améliorer leur qualité en éliminant la végétation sénescente et la litière et, par ce moyen, favorisent les repousses des graminées ;

- d'après des essais réalisés dans les Llanos de Colombie (11), le pâturage après feux, à faible charge, permet des gains de poids annuels par hectare plus importants (de deux à trois fois), comparativement à une gestion sans feux (tableau I).

C'est une pratique généralisée sur les 55 millions d'hectares de prairies du bassin de l'Orénoque au nord du continent sud-améri- 
Tableau I

Comparaison des gains de poids de bovins sur pâturages naturels des Llanos de Colombie et de l'Adamaoua au Cameroun exploités traditionnellement avec ou sans feu de saison sèche selon différentes charges et sur différents types de sols

\section{Type de gestion} et type de sol

\section{Chargement moyen En kg poids vif/ha}

\section{Gains moyens quotidiens} En $g$ poids vif/tête/j

$\begin{array}{cc}\text { Saison des } & \text { Saison sèche } \\ \text { pluies }(235 \mathrm{j}) & (130 \mathrm{j})\end{array}$

\section{Gains annuels}

En $\mathrm{kg}$ poids vif/ En $\mathrm{kg}$
tête de $250 \mathrm{~kg}$ poids vif/ha
(U bt)

\section{Amérique du Sud (Llanos de Colombie) *}

Traditionnelle sans feu,

libre parcours sur sol argileux

40

66

100

Feu en saison sèche, libre parcours

+ minéraux sur sol argileux

\section{0}

66

100
210

270

150

260

310

230
$-160$

$-190$

$-250$

$+110$

$-50$

$-180$
28

38

2

75

67

31
4,5

9,9

0,8

12,0

17,4

12,4

\begin{tabular}{|c|c|c|c|c|c|}
\hline \multicolumn{6}{|c|}{$\begin{array}{l}\text { Afrique (Adamaoua au Cameroun) ** } \\
\text { Vaine pâture sans feu }\end{array}$} \\
\hline Sol argileux & 350 & 600 & -129 & 102 & 142,8 \\
\hline Sol sableux & 175 & 600 & -187 & 54 & 37,7 \\
\hline \multicolumn{6}{|c|}{ Rotation et feu de saison sèche } \\
\hline Sol argileux & 450 & +547 & -12 & 147 & 267,6 \\
\hline Sol sableux & 350 & +420 & -132 & 73 & 102,2 \\
\hline
\end{tabular}

* D'après Paladines O., Leal J., 1979, Pasture management and productivity in the Llanos Orientales, CIAT

*** D'après Rippstein G., 1985, Etudes et synthèses n 14 , Iemvt

cain où pâturent 8 millions de têtes de bovins. Dans ces prairies, on retrouve donc les mêmes préoccupations des éleveurs et les mêmes moyens de gestion que ceux d'Afrique (2) ou d'Australie (8).

En Afrique, sur pâturage naturel et dans des conditions de pluviométrie assez proches de celles des Llanos, les gains moyens quotidiens (gmq) y sont très supérieurs (12). En effet, sur les savanes arbustives du plateau de l'Adamaoua au Cameroun (tableau I), dans des systèmes extensifs et quelque peu améliorés par des rotations, mais sans complémentation, les gmq sont de 600 à $700 \mathrm{~g}$ par tête par jour avec feu et de 400 à $500 \mathrm{~g}$ sans feu pendant les cinq à six mois de saison des pluies $(\mathrm{Sp})$. En saison sèche $(\mathrm{Ss})$, au contraire, les pertes de poids peuvent atteindre $200 \mathrm{~g}$ par tête par jour, même avec une complémentation d'environ $100 \mathrm{~g}$ de matières azotées totales (Mat) pour $100 \mathrm{~kg}$ de poids vif par jour quelle que soit la charge animale ou le système d'exploitation du pâturage.

Pour améliorer la productivité des pâturages naturels des savanes de Colombie et du Venezuela avec un minimum d'intrants et sans autre intervention sur la végétation naturelle que la modification de la gestion des feux, des rythmes de rotation et du chargement, les auteurs ont élaboré et testé, en station, en petites parcelles puis avec des animaux, différents modèles de gestion.

\section{MATERIEL ET METHODES}

\section{Le site}

Les expérimentations ont été réalisées de 1991 à 1997 sur le Centre de recherches de Corpoica (Corporation Colombiana de Investigación Agropecuaria)/Ciat (Centro Internacional de Agricultura Tropical) de Carimagua (latitude $4^{\circ} 37^{\prime} \mathrm{N}$, longitude $71^{\circ} 19^{\prime} \mathrm{O}$ et $175 \mathrm{~m}$ d'altitude), soit au centre géographique des Llanos de Colombie dans le bassin du fleuve Orénoque.

\section{Climat}

Le climat y est tropical humide avec $2329 \mathrm{~mm}$ de précipitations moyennes annuelles (moyenne sur 23 ans ; coefficient de variation : 13,5 p. 100) réparties sur huit mois de saison des pluies (de mars à mi-décembre) et une saison sèche de quatre mois qui n'est jamais « écologiquement sèche ». Les quelques pluies permettent de maintenir une végétation active pendant cette saison.

La température moyenne de $26,8{ }^{\circ} \mathrm{C}$ est remarquablement stable au cours de l'année, mais les amplitudes journalières sont variables : la moyenne des températures maximales journalières atteint $33,4{ }^{\circ} \mathrm{C}$ en février-mars (la température maximale est de $36{ }^{\circ} \mathrm{C}$ en mars) et la moyenne des températures minimales journalières est de $21,5^{\circ} \mathrm{C}$ en janvier et en juillet (la température minimale est de $18{ }^{\circ} \mathrm{C}$ ). L'humidité relative mensuelle varie de 64 à 73 p. 100 en Ss et de 80 à 95 p. 100 en Sp. L'évaporation en bac est en moyenne de $220 \mathrm{~mm}$ par mois en Ss et de $120 \mathrm{~mm}$ en Sp.

\section{Sols}

Les expérimentations ont été réalisées sur deux types de sols représentatifs des Llanos colombiens : des sols ferrallitiques argilolimoneux (Al) (Oxisols dans la classification américaine) et des sols ferrallitiques sablo-argileux ( $\mathrm{Sa}$ ) dont les caractéristiques sont présentées au tableau II. Les premiers sont représentatifs des plateaux des hautes plaines planes (Altillanura plana) qui occupent environ 3,5 millions d'hectares dans les Llanos de Colombie (3); les seconds sont proches des sols des zones des «plaines ondulées et des collines » (Serrania) qui occupent 6,4 millions d'hectares dans les Llanos de Colombie. On remarque des différences importantes dans la texture de ces sols : un $\mathrm{pH}$ généralement plus bas 
pour les sols $\mathrm{Al}$, des différences significatives pour les teneurs en aluminium, calcium, potassium, azote total et disponible, pour le rapport carbone/azote et pour le phosphore utilisable.

\section{Végétation}

Bien que les conditions climatiques soient celles de la forêt équatoriale sempervirente ou de la savane arborée d'Afrique, les conditions édaphiques des Llanos font que la végétation naturelle des interfluves est une prairie ou une savane basse, pratiquement sans ligneux (18).

\section{Tableau II}

Caractéristiques des principaux sols des hautes plaines planes et collines (Altillanura et Serrania) des Llanos

\begin{tabular}{|c|c|c|}
\hline \multirow[b]{2}{*}{ Caractéristiques } & \multicolumn{2}{|c|}{ Sols $(0-20 \mathrm{~cm})$} \\
\hline & $\begin{array}{l}\text { Ferrallitique } \\
\text { Al }\end{array}$ & $\begin{array}{l}\text { Ferrallitique } \\
\text { Sa }\end{array}$ \\
\hline Argile (\%) & 36 & 17 \\
\hline Sable (\%) & 24 & 65 \\
\hline M atière organique $(\%)$ & 3,4 & 0,89 \\
\hline $\mathrm{pH}\left(\mathrm{H}_{2} \mathrm{O}\right)$ & 4,5 & 5,1 \\
\hline Aluminium (meq/100 g) & 2,3 & 0,7 \\
\hline Calcium $(\mathrm{meq} / 100 \mathrm{~g})$ & 0,09 & 0,13 \\
\hline Magnésium (meq/100 g) & 0,06 & 0,08 \\
\hline Potassium (meq/100 g) & 0,08 & 0,03 \\
\hline Saturation aluminium (\%) & 88 & 77 \\
\hline Azote total (mg/kg) & 1254 & 339 \\
\hline $\begin{array}{l}\text { Azote disponible }(\mathrm{mg} / \mathrm{kg}) \\
\left(\mathrm{NO}_{3}-\mathrm{N}+\mathrm{NH}_{4}-\mathrm{N}\right)\end{array}$ & $\begin{array}{c}2,57 \\
+7,36\end{array}$ & 6,23 \\
\hline Carbone/azote & 19,8 & 15,5 \\
\hline $\begin{array}{l}\text { Phosphore utilisable } \\
\text { (mg/kg) (Bray II) }\end{array}$ & 3,9 & 2,0 \\
\hline
\end{tabular}

$\mathrm{Al}=$ argilo-limoneux $; \mathrm{Sa}=$ sablo-argileux
Les ligneux sont surtout présents le long des cours d'eau (forêtgalerie). La pauvreté chimique des sols des Llanos et la présence d'une nappe phréatique proche du sol expliquent la physionomie de la végétation et sa faible productivité (18). La prairie est dominée essentiellement par des graminées et des cypéracées. Les dicotylédones herbacées et particulièrement les légumineuses y jouent un rôle très secondaire. Les principales espèces des deux types de sols ( $\mathrm{Al}$ et $\mathrm{Sa}$ ) et leurs contributions spécifiques moyennes des zones étudiées sont rapportées dans le tableau III. Les différences d'espèces et les contributions spécifiques des espèces communes sont souvent importantes.

\section{Etude des repousses après feu en mini-parcelles}

\section{Dispositif et facteurs étudiés}

Avant d'entreprendre des essais dans un dispositif avec des animaux, une première phase d'essais a consisté à mesurer sur trois années en petites parcelles de $16 \mathrm{~m}^{2}(4 \mathrm{x} 4 \mathrm{~m})$, la productivité, la composition botanique, la dynamique de la végétation et la composition chimique des repousses après feu, sur les deux types de sols des Llanos ( $\mathrm{Al}$ et $\mathrm{Sa}$ ), dans un dispositif en blocs avec quatre répétitions.

Le dispositif combinait :

- la saison des brûlages (Ss et $\mathrm{Sp}$ ) ;

- la durée des mises en défens après feu initial de « remise à zéro de la végétation » correspondant à des périodes respectives de 8 , 12 et 16 mois de repos avant un nouveau feu et une répétition de parcelles qui ne sont pas brûlées (témoins sans feu). Ceci devait permettre de déterminer le meilleur temps de repos nécessaire pour obtenir une biomasse suffisante pour des feux efficaces ;

- la récolte respective des repousses toutes les 1,2, 4 et 8 semaines (coupes à $5 \mathrm{~cm}$ du sol pour simuler des prélèvements par les animaux) pour des mesures de productivité (le poids sec des prélèvements a été déterminé après passage à l'étuve à $70{ }^{\circ} \mathrm{C}$ jusqu'à poids constant) ;

- la dynamique de la végétation étudiée par des mesures de la présence des espèces, avant chaque coupe, selon la méthode des points quadrats alignés $(5,6)$, sur les deux diagonales des parcelles.

\section{Tableau III}

Principales espèces des pâturages naturels et leur contribution spécifique (Cs) dans les deux principaux types de sols des Llanos

\section{Sur sol argilo-limoneux}

Sur sol sablo-argileux

\begin{tabular}{lccr}
\hline Espèces & Cs (\%) & Espèces & Cs (\%) \\
\hline $\begin{array}{l}\text { Graminées } \\
\quad \text { Trachypogon vestitus }\end{array}$ & Graminées & Paspalum pectinatum & 13,8 \\
$\quad$ Axonopus purpusii & 41 & Schizachyrium hirtiflorum & 12,7 \\
$\quad$ Paspalum pectinatum & 20 & Axonopus purpusii & 10,5 \\
$\quad$ Andropogon leucostachyus & 11 & Trachypogon vestitus & 10,2 \\
$\quad$ Schizachyrium hirtiflorum & 5 & M esosetum pittieri & 9,1 \\
$\quad$ Trasya petrosa & 5 & Leptocoryphium lanatum & 6,5 \\
Dicotylédones diverses & 2 & Autres graminées & 13,8 \\
Cypéracées & 3 & Dicotylédones & 0,1 \\
Légumineuses & 0,5 & Cypéracées & Rhynchospora podosperma \\
& & Rhynchospora confinis & 13,2 \\
& & & 9,2
\end{tabular}




\section{Valeur nutritive du tapis herbacé}

Les teneurs en matières azotées totales et la digestibilité in vitro de la matière organique (Dmo) ont été déterminées selon les méthodes utilisées dans les laboratoires du Ciat $(17,19)$.

\section{Essais sur grandes parcelles avec animaux}

Au cours d'une deuxième phase, des essais de pâturage ont été réalisés selon deux systèmes (tableau IV ; figures 1 et 2).

\section{Dispositif 1}

Ce dispositif (ou système 1) a été appliqué à un bloc clôturé de 24 ha de 1991 à 1993, réduit à 16 ha par la suite, divisé en quatre parcelles équivalentes. Les parcelles, non clôturées, ont été brûlées successivement avec un intervalle de quatre mois entre deux parcelles. Il a été ainsi mis à la disposition des animaux de nouvelles repousses de 0 à 4 mois sur un quart du dispositif. Les animaux avaient donc le choix de repousses de différents âges variant de 0 à 16 mois (figure 1). Ce dispositif a été mis en place sur sol Sa et sur sol Al et les mesures ont été faites de décembre 1991 à février 1997

\section{Dispositif 2}

Ce dispositif (ou système 2) était composé de deux blocs clôturés contigus. Chaque bloc de 25 ha était divisé en 10 parcelles de même dimension non clôturées, mais entourées chacune par un pare-feu de deux mètres de largeur. Chaque parcelle a été brûlée tous les 16 mois et le repos avant exploitation de la parcelle récemment brûlée a été d'un mois car l'objectif a été de renouveler, chaque mois, l'offre de jeune fourrage. Les parcelles d'un même bloc n'ayant pas été clôturées, les animaux ont disposé en fait de fourrage d'âge variable compris entre $0-15$ mois et $1-16$ mois (figure 2).

L'itinéraire technique de ce système 2 a consisté à effectuer mensuellement le brûlis d'une parcelle de 2,5 ha en Sp (maidécembre), le bloc contenant la parcelle qui venait d'être brûlée a été laissé en défens (hors pâture) pendant un mois. Les animaux, pendant ce temps-là, ont pâturé le bloc qui avait des repousses de $1,3,5,7 \ldots 15$ mois. Chaque mois, les animaux ont été déplacés d'un bloc à l'autre. En Ss, comme la croissance du tapis herbacé a été ralentie, deux parcelles du même bloc ont été brûlées simultanément tous les mois, d'où les dix parcelles dans chaque bloc. Ainsi une partie du dispositif (1/20 en Sp et $2 / 20$ en Ss) a été brûlée mensuellement et successivement. Les animaux ont pâturé alternativement sur deux blocs après un mois de mise en repos du bloc. Chaque parcelle a donc bien été brûlée tous les 16 mois. Ce système a été réalisé sur sol $\mathrm{Al}$ seul et sur une période allant de 1994 à 1996.

\section{Animaux et charges}

Les essais ont été réalisés avec des bovins mâles en croissance de race métis (Criollo x zébu Brahman) dont le poids moyen a été de $186 \mathrm{~kg}$ en début d'essai. Il y a eu quatre animaux dans le système 1 et dix dans le système 2 . Ils ont reçu une complémentation minérale à volonté et ont eu un accès permanent à des abreuvoirs. Les animaux ont été pesés mensuellement pour calculer leurs gains de poids : gains moyens quotidiens, gains mensuels, saisonniers, annuels et sur la totalité de l'essai.

Les charges animales ont été déterminées suite aux mesures de productivité de la végétation en petites parcelles. De 1991 à 1993 pour le système 1 sur sol $\mathrm{Sa}$, une charge considérée comme modérée (6 ha/animal) a été appliquée la première année, puis, compte tenu de l'importance des refus, une charge considérée comme forte (4 ha/animal) a été adoptée à partir de la seconde année en diminuant la surface exploitée. Elle a été comparée à un même dispositif sur sol $\mathrm{Al}$ chargé également à 4 ha par animal considéré ici comme une charge modérée.

Dans un nouvel essai mis en place dès 1994, dans le système 1, sur sol $\mathrm{Al}$, trois charges (forte, moyenne et faible, soit respectivement 2,4 et 6 ha par animal) ont été comparées. Le système 1 a été comparé au système 2 sur sol $\mathrm{Al}$ chargé à 4 ha par animal.

Tableau IV

Protocole et facteurs étudiés

\begin{tabular}{|c|c|c|}
\hline Facteurs & $\begin{array}{l}\text { Système } 1 \\
\text { Traditionnel amélioré en } 4 \text { quartiers } \\
\text { et feux séquentiels tous les } 4 \text { mois }\end{array}$ & $\begin{array}{l}\text { Système } 2 \\
\text { Amélioré avec rotation sur } 2 \text { blocs } \\
\text { et feux séquentiels tous les mois }\end{array}$ \\
\hline Type de sol & $\begin{array}{l}\text { Argilo-limoneux (Al) } \\
\text { Sablo-argileux (Sa) }\end{array}$ & Argilo-limoneux \\
\hline $\mathrm{Nb}$. de blocs & 1 & 2 \\
\hline Nb. de parcelles/bloc & 4 & 10 \\
\hline Fréquence des brûlis de chaque parcelle & Tous les 16 mois & Tous les 16 mois \\
\hline $\begin{array}{l}\text { Nb. de parcelles brûlées et mises à disposition } \\
\text { des animaux et fréquence }\end{array}$ & Une tous les 4 mois & $\begin{array}{l}\text { Une tous les mois en saison des pluies } \\
\text { Deux tous les mois en saison sèche }\end{array}$ \\
\hline Repos après brûlage avant exploitation & Pâture juste après brûlis & 1 mois \\
\hline Rotation : type/fréquence & Non & Alternée sur 2 blocs \\
\hline \multicolumn{3}{|l|}{ Chargement } \\
\hline 1991 & $\begin{array}{l}\text { Sa : } 6 \text { ha/tête (charge modérée) } \\
\text { Al : } 4 \text { ha/tête/an (charge modérée) }\end{array}$ & Pas de comparaison \\
\hline 1992-1993 & $\begin{array}{c}\text { Sa : } 4 \text { ha/tête/an (charge forte) } \\
\text { Al : } 4 \text { ha/tête/an (charge modérée) }\end{array}$ & Pas de comparaison \\
\hline 1994-96 & $\begin{array}{c}\text { Al : } 2 \text { ha/tête/an (charge forte) } \\
4 \text { ha/tête/an (modérée) } \\
6 \text { ha/tête/an (faible) }\end{array}$ & Al : 4 ha/tête/an (modérée) \\
\hline
\end{tabular}




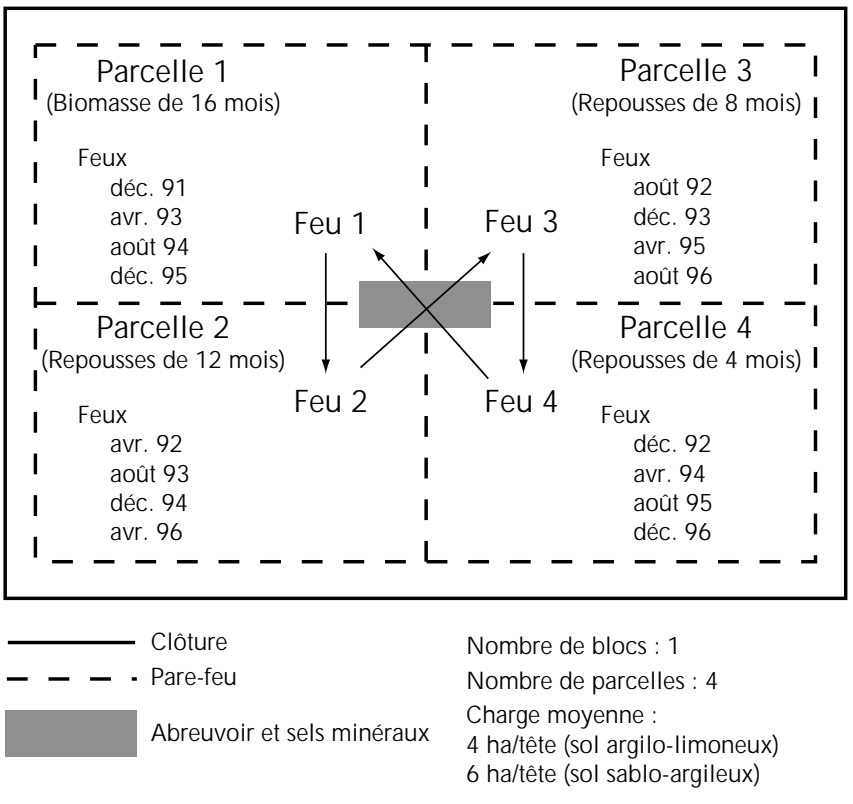

Figure 1 : dispositif 1 . Gestion traditionnelle améliorée (en quartiers) des pâturages avec mise en repos de 16 mois de la végétation et rotation des feux tous les quatre mois.

\section{Mesures et observations sur les pâturages}

Les mesures et les observations ont concerné la productivité et la composition des pâturages, le comportement alimentaire et les gains de poids des animaux.

La production végétale aérienne après feu sans pâture a été mesurée dans des mises en défens (cinq cages de 1,5 x 1,5 x 1,5 m). Des coupes ont été effectuées tous les mois à $5 \mathrm{~cm}$ du sol dans cinq placettes de $1 \mathrm{~m}^{2}$ chacune, suivies de la pesée de la biomasse verte, puis du séchage à l'étuve à $70^{\circ} \mathrm{C}$ jusqu'à poids constant pour déterminer la matière sèche (Ms). Avant séchage, les espèces les plus importantes de la végétation récoltée ont été triées et identifiées afin d'obtenir la production par espèce.

La composition botanique des régimes alimentaires et le comportement des animaux au pâturage ont été observés avec les méthodes suivantes :

- par comptage, selon la méthode des points quadrats alignés de Daget et Poissonet (6), le long de transects (cinq transects de $20 \mathrm{~m}$ ), avant l'entrée des animaux dans la parcelle et juste après leur sortie (ces mesures permettent de prendre en compte non seulement les espèces pâturées mais aussi les espèces arrachées);

- par l'observation visuelle directe du tapis herbacé à proximité des animaux (relevé des espèces fraîchement consommées).

Le comportement des animaux au pâturage a été observé visuellement durant plusieurs mois $(1,4)$. Leur position dans le bloc exploité a été notée tous les quarts d'heure, par le relevé du numéro de la parcelle pâturée et par leurs prises d'aliments. Ces observations ont été réalisées uniquement de jour pour des raisons de sécurité et à un rythme de trois jours par semaine pendant six mois (de mai à novembre).

La valeur pastorale a été établie par la combinaison des facteurs productivité, valeur nutritive et appétence de chaque espèce et par l'établissement, pour chaque espèce, d'un indice individuel de valeur pastorale (Ivp). Combiné à la contribution de chaque espèce au pâturage, il permet de calculer, pour chaque type de pâturage, un indice dénommé « valeur pastorale » $(\mathrm{Vp})(13)$.

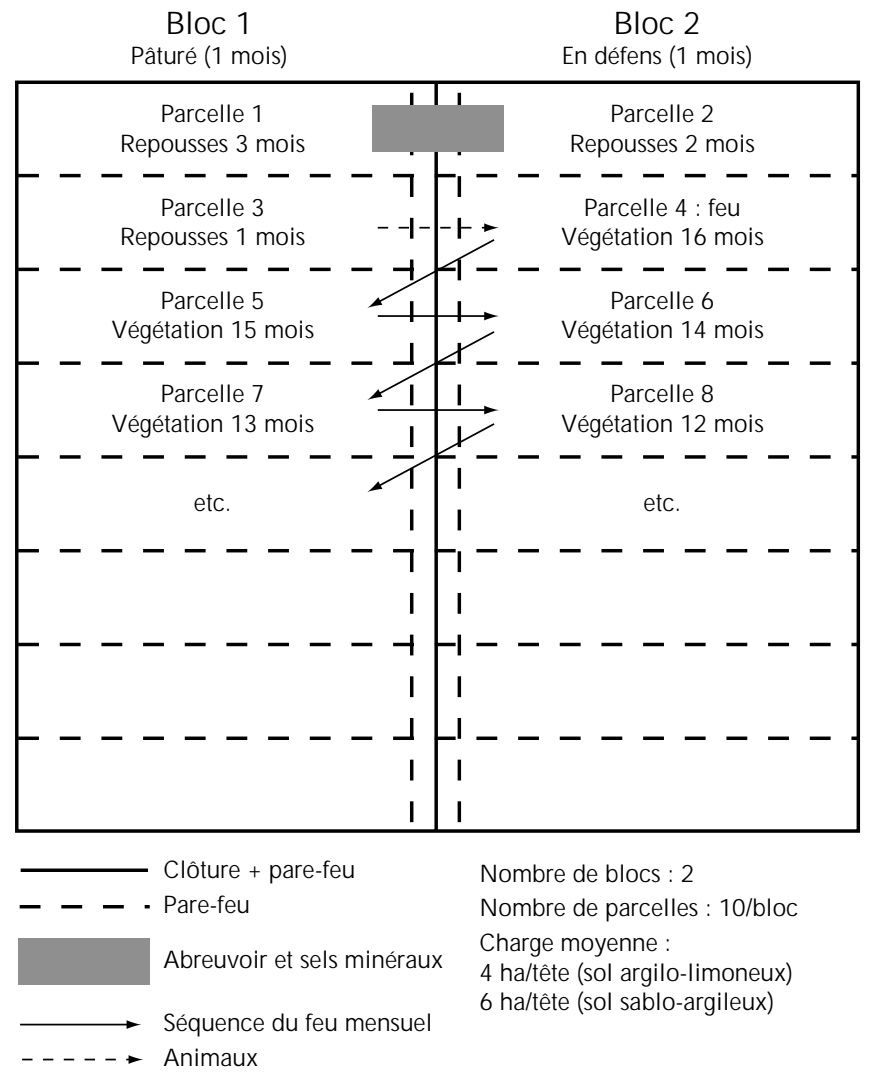

Figure 2 : dispositif 2 . Gestion améliorée des pâturages avec mise en repos de 16 mois de la végétation et rotation des feux tous les mois.

\section{RESU LTATS ET DISCUSSIO N}

\section{Productivité, valeur alimentaire et valeur pastorale des formations naturelles}

\section{Productivité et valeur nutritive}

Les mesures de productivité (tableau V) sur petites parcelles ont montré qu'en saison des pluies la productivité journalière des jeunes repousses de la première semaine sur sols $\mathrm{Al}$ et des deux premières semaines sur sols $\mathrm{Sa}$ ( $5 \mathrm{~kg}$ de $\mathrm{Ms} / \mathrm{ha} / \mathrm{j}$ environ) a été inférieure de moitié à celle des repousses de quatre semaines et plus $(10 \mathrm{~kg}$ de $\mathrm{Ms}$ environ). Pour ces dernières, il n'y a pas eu de différence entre les types de sols malgré la plus faible fertilité théorique des sols $\mathrm{Sa}$. Au contraire, en saison sèche, la productivité journalière sur sol $\mathrm{Sa}$ a été en moyenne supérieure à celle des sols $\mathrm{Al}$ (respectivement 7,6 et $4,8 \mathrm{~kg} \mathrm{Ms} / \mathrm{ha} / \mathrm{j}$ ). Elle a été deux fois supérieure pour des repousses d'une et de deux semaines (respectivement 8,6-9,0 et 4,0-5,9 kg $\mathrm{Ms} / \mathrm{ha} / \mathrm{j}$ sur sol $\mathrm{Al}$ ). Elle a été maximale dès les premières semaines dans les deux situations et a diminué en fin de cycle sur les sols Sa.

Ainsi, en Ss, la réserve d'eau utilisable (différence entre capacité au champ et le point de flétrissement) a semblé plus importante dans les sols Sa que dans les sols Al et le manque d'eau et de nutriments n'a paru intervenir qu'en fin de cycle sur la vitesse de croissance pour les sols $\mathrm{Sa}$.

Concernant la valeur nutritive du tapis herbacé dans son intégralité, sans tenir compte de l'appétence des espèces, pour les deux types de pâturages en $\mathrm{Sp}$, une teneur considérée moyenne en Mat (supérieure à 7 p. 100 de la Ms) a été observée durant les deux et les quatre premières semaines de repousse respectivement sur sols 
Sa et Al, suivie d'une rapide diminution. En Ss, les teneurs en Mat des repousses ont été moins élevées qu'en Sp sur sol Al, alors qu'elles ont été de valeur égale à celle de la $\mathrm{Sp}$ sur sol $\mathrm{Sa}$.

Les Dmo ont diminué rapidement en Sp sur les deux types de sols après deux à quatre semaines de végétation alors qu'elles sont restées constantes en Ss, tout au moins jusqu'à huit semaines. Les Dmo ont été significativement supérieures en Ss par rapport à la $\mathrm{Sp}$, surtout sur sol Al et pour les repousses les plus âgées des sols Sa.

\section{Valeur pastorale}

L'observation des animaux au pâturage et des espèces appétées a permis de déterminer l'appétence des principales espèces. Celle-ci, combinée à la productivité et à la valeur nutritive des repousses de
4,8 et 16 semaines pour chaque espèce (tableau VI) a permis de déterminer leur indice spécifique de valeur pastorale qui, multiplié par la contribution spécifique $(\mathrm{Cs})$ de chacune de ces espèces au pâturage, permet d'en connaître la valeur pastorale.

C'est ainsi qu'en prenant les Cs observées pour les pâturages sur sols $\mathrm{Al}$ et $\mathrm{Sa}$ (tableau III) la Vp de ces deux formations a pu être calculée. Pour des repousses de 4,8 et 16 semaines, elle a été respectivement de 58,9,63,5, et 44,7 p. 100 pour les pâturages sur sol $\mathrm{Al}$, et de 43,5, 48,4 et 38,2 p. 100 sur sol Sa.

On constate donc que les pâturages sur sols $\mathrm{Al}$ ont eu une meilleure valeur pastorale que ceux sur sols $\mathrm{Sa}$, quel qu'ait été l'âge des repousses. L'importance des différences de composition

\section{Tableau V}

Productivité et valeur nutritive des repousses après feu des prairies naturelles des zones planes sur sol argilo-limoneux (Altillanura plana) et des collines (Serrania) sur sol sablo-argileux des Llanos de Colombie

$\begin{aligned} & \text { Age des repousses Productivité } \\ & \text { (semaines) } \\ & \text { (kg Ms/ha/jour) }\end{aligned}$

Sp Ss

1

1

4

8

Moyenne

)

$5,9^{\mathrm{b} *} \quad 4,0^{\mathrm{a}} \quad 10,4^{\mathrm{a}}$

$8,6^{\mathrm{b}} \quad 6,6^{\mathrm{a}}$

$\begin{array}{llll}10,4^{\mathrm{a}} & 5,9^{\mathrm{a}} & 8,6^{\mathrm{b}} & 6,6^{\mathrm{a}} \\ 10,6^{\mathrm{a}} & 4,4^{\mathrm{a}} & 8,4^{\mathrm{b}} & 6,2^{\mathrm{a}}\end{array}$

$8,9^{\mathrm{ab}} \quad 5,0^{\mathrm{a}}$

$5,7^{c} \quad 5,9^{a}$
Sol argilo-limoneux (AI)

1

* Les chiffres suivis de la même lettre (dans les colonnes) ne sont pas significativement différents $(\mathrm{P}<0,05)$

$\mathrm{Ms}=$ matière sèche $; \mathrm{Sp}=$ saison des pluies $; \mathrm{Ss}=$ saison sèche $;$ Mat = matières azotées totales $;$ Dmo = digestibilité in vitro de la matière organique

\section{Sol sablo-argileux (Sa)}

Productivité
(kg Ms/ha/jour)

Sp Ss

$\mathrm{Sp}^{\text {Mat }}$ Ss $\quad$ Sp ${ }^{\text {Dmo }}$ Ss

$44,6^{\mathrm{a}}-$

$43,6^{\mathrm{a}} \quad 48,1^{\mathrm{a}}$

$40,3^{\mathrm{b}} \quad 48,8^{\mathrm{a}}$

$33,7^{c} \quad 45,5^{b}$

$40,6 \quad 47,5$

\section{(\% de Ms)}

\begin{tabular}{|c|c|c|c|}
\hline \multicolumn{2}{|c|}{ Mat } & \multicolumn{2}{|c|}{ Dmo } \\
\hline Sp & Ss & Sp & Ss \\
\hline $8,9^{a}$ & - & $44,5^{a}$ & - \\
\hline $7,7^{b}$ & $8,8^{a}$ & $43,6^{a}$ & $44,1^{\mathrm{a}}$ \\
\hline $6,2^{c}$ & $6,0^{b}$ & $38,0^{b}$ & $45,1^{\circ}$ \\
\hline $4,7^{d}$ & $5,6^{d}$ & $31,2^{c}$ & $44,9^{a}$ \\
\hline 6,9 & 6,8 & 39,3 & 44,7 \\
\hline
\end{tabular}

\section{Tableau VI}

Indices de la valeur pastorale des principales espèces des prairies naturelles des Llanos de Colombie

\begin{tabular}{|c|c|c|c|c|c|c|c|c|c|c|c|c|}
\hline \multirow{3}{*}{ Espèces } & \multicolumn{12}{|c|}{ Age des repousses } \\
\hline & \multicolumn{4}{|c|}{4 semaines } & \multicolumn{4}{|c|}{8 semaines } & \multicolumn{4}{|c|}{16 semaines } \\
\hline & (1) & (2) & (3) & (4) & (1) & (2) & (3) & (4) & (1) & (2) & (3) & (4) \\
\hline Andropogon bicornis & 0 & 1 & 1 & 0,7 & 2 & 1 & 1 & 1,3 & 1 & 1 & 1 & 1,0 \\
\hline Leptocoryphium Ianatum & 1 & 1 & 2 & 1,3 & 1 & 1 & 2 & 1,3 & 1 & 2 & 2 & 1,7 \\
\hline Schizachyrium hirtiflorum & 0 & 1 & 3 & 1,3 & 2 & 2 & 3 & 2,3 & 2 & 3 & 1 & 2,0 \\
\hline Andropogon leucostachyus & 3 & 1 & 2 & 2,3 & 3 & 1 & 2 & 2,0 & 2 & 1 & 1 & 1,3 \\
\hline Paspalum pectinatum & 2 & 3 & 3 & 2,3 & 2 & 3 & 1 & 2,0 & 1 & 3 & 1 & 1,7 \\
\hline Axonopus purpusii & 2 & 3 & 3 & 2,7 & 3 & 3 & 3 & 3,0 & 2 & 2 & 2 & 2,0 \\
\hline Trachypogon vestitus & 3 & 2 & 3 & 2,7 & 3 & 3 & 3 & 3,0 & 1 & 2 & 1 & 1,3 \\
\hline O tachyrium versicolor & 1 & 1 & 2 & 1,3 & 4 & 2 & 2 & 2,7 & 3 & 1 & 2 & 2,0 \\
\hline Autres graminées & 1 & 1 & 3 & 1,7 & 2 & 1 & 2 & 1,7 & 1 & 2 & 1 & 1,3 \\
\hline Légumineuses diverses & 2 & 1 & 3 & 2,0 & 3 & 2 & 3 & 2,7 & 3 & 1 & 3 & 2,3 \\
\hline Cypéracées diverses & 0,5 & 1 & 1 & 0,8 & 1 & 1 & 1 & 1,0 & 1 & 1 & 1 & 1,0 \\
\hline Dicotylédones divers & 0,5 & 1 & 1 & 0,8 & 0,5 & 1 & 0 & 0,5 & 0,5 & 1 & 0 & 0,5 \\
\hline
\end{tabular}

(1) Appétence : $0=$ non appété $; 1=$ peu appété ; $2=$ moyennement appété ; $3=$ appété ; $4=$ très appété

(2) Productivité : $0=$ sans production $; 1=$ non productive $; 2=$ peu productive $; 3=$ productive $; 4=$ très productive

(3) Valeur alimentaire : $0=$ nulle ; $1=$ médiocre ; $2=$ moyenne ; $3=$ bonne ; $4=$ très bonne

(4) Indice de valeur pastorale $=$ moyenne arithmétique de (1), (2) et (3) 
botanique des pâturages et des contributions spécifiques des espèces a ainsi été établie.

\section{Performances des bovins sur les prairies naturelles gérées par les feux}

Ces essais préliminaires de productivité et de valeur du pâturage ont fait ressortir qu'il était nécessaire d'exploiter le pâturage après au moins deux à quatre semaines de repos après feu, que la charge de 8 à 10 ha par animal traditionnellement appliquée par les éleveurs pouvait être augmentée à 4 ou 6 ha par animal et qu'il était nécessaire de moduler la charge en fonction du type de sol et donc de la formation végétale. Ces observations ont été testées ou confirmées avec des animaux dans différents systèmes.

\section{Exploitation des pâturages naturels de différents types de sols selon le système 1}

Les gains de poids mensuels cumulés durant deux années pour le système de gestion « traditionnelle améliorée », en quatre quartiers et feux séquentiels tous les quatre mois (système 1) sur les deux types de prairies naturelles (sols $\mathrm{Al}$ et $\mathrm{Sa}$ ) sont représentés à la figure 3. D'août 1991 à juin 1992 (figure 3, tableau VII), les animaux ont eu des gains de poids égaux bien que cette évolution ait été plus irrégulière sur la prairie des sols Sa que sur celle des sols Al. Même si, en début d'essai, soit d'août à décembre 1991 (tableau VII), les gmq ont été un peu plus importants sur sol $\mathrm{Al}$ que sur sol Sa (respectivement +228 et $+207 \mathrm{~g}$; différence non significativement différente), ensuite de décembre à juin, les pertes de poids ont été plus importantes sur sol $\mathrm{Al}$, tous les animaux ayant atteint le même poids en juin 1992.

A partir de juin-juillet 1992, les gains de poids ont divergé rapidement pour atteindre, en septembre 1993, un écart de plus de $90 \mathrm{~kg}$ en faveur des animaux sur sol Al.

Cette évolution divergente des courbes de gains de poids des animaux a coïncidé avec une modification du chargement. En effet, durant la première période (d'août 1991 à juin 1992), la charge a été de 4 ha par animal sur sol $\mathrm{Al}$ et de 6 ha par animal sur sol Sa, alors qu'à partir de juin les chargements ont été identiques pour les deux sites, soit 4 ha par animal, correspondant à une charge modérée sur sol $\mathrm{Al}$ et à une charge forte sur sol $\mathrm{Sa}$.

Par ailleurs, durant les Ss des deux années de mesure, sur les deux sites $\mathrm{Al}$ et $\mathrm{Sa}$, les animaux ont perdu du poids la première année (gmq respectivement de - 57 et - $10 \mathrm{~g}$ ) et n'ont eu que de faibles gains de poids journaliers la seconde année (respectivement +35 et $+21 \mathrm{~g}$ ), ceci malgré la présence de jeunes repousses après les feux de décembre de 1991 et 1992. Si les valeurs fourragères de ces repousses ont été excellentes après les feux, en revanche les quantités disponibles ont probablement été insuffisantes.
La différence de gmq des animaux entre les deux sites (différence significative pour toutes les saisons dès décembre 1991) peut s'expliquer, en partie, par la différence de la valeur azotée du pâturage (tableau V), mais il semble surtout que la différence de composition botanique, elle-même due aux différences de conditions édaphiques, ait eu une plus grande importance.

En effet, sur sol Sa, le tapis herbacé était composé d'une proportion importante de cypéracées (plus de 22 p. 100) (tableau III), très peu appétées et de très faible valeur (Ivp $=0,5$ ) et de graminées peu appétées comme Schizachyrium hirtiflorum (Ivp = 1,3), alors que sur sol Al, Trachypogon vestitus et Axonopus purpusii, qui ont produit plus de 60 p. 100 de la biomasse, ont été très appétées et ont eu l'Ivp le plus élevé des espèces présentes $(2,7)$.

En conséquence, la valeur pastorale moyenne de 55,7 p. 100 (moyenne des repousses de 4, 8 et 16 semaines) pour les pâturages des sols Al et seulement de 43,4 p. 100 pour ceux des sols Sa peut mieux expliquer ces différences de gains de poids des animaux que la différence de productivité et/ou la valeur alimentaire de ces

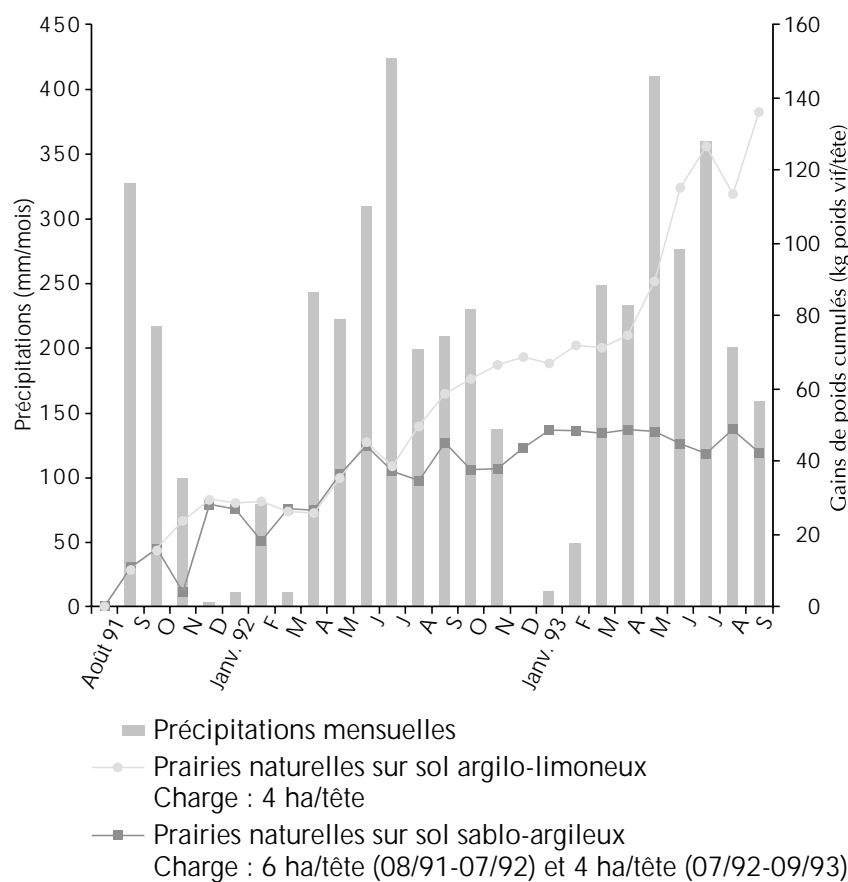

Figure 3 : comparaison de l'évolution des gains de poids de bovins sur deux types de prairies naturelles des Llanos de Colombie exploités selon un système de gestion par les feux séquentiels en rotation tous les quatre mois.

Tableau VII

Gains de poids quotidiens (en $\mathrm{g}$ ) de jeunes bovins sur deux types de pâturages des Llanos de Colombie dans un système de gestion traditionnelle améliorée par les feux (système 1)

\begin{tabular}{|c|c|c|c|c|c|c|c|c|}
\hline Type de sol & $\begin{array}{c}\text { Nb. } \\
\text { d'animaux }\end{array}$ & $\begin{array}{c}\text { Fin Sp } \\
1991 \\
\text { (août-déc.) }\end{array}$ & $\begin{array}{c}\text { Ss } \\
\text { 1991-92 } \\
\text { (déc-avr.) }\end{array}$ & $\begin{array}{c}\text { Sp } \\
1992 \\
\text { (avr.-déc.) }\end{array}$ & $\begin{array}{c}\text { Ss } \\
\text { 1992-93 } \\
\text { (déc.-mars) }\end{array}$ & $\begin{array}{c}\text { Début Sp } \\
1993 \\
\text { (mars-sept.) }\end{array}$ & $\begin{array}{r}\text { Sp + Ss } \\
1992-93\end{array}$ & $\begin{array}{c}\text { Total } \\
\text { essai } \\
\text { (25 mois) }\end{array}$ \\
\hline $\begin{array}{l}\text { Al } \\
\text { Sa }\end{array}$ & $\begin{array}{l}10 \\
10\end{array}$ & $\begin{array}{l}+228^{a *} \\
+207^{a}\end{array}$ & $\begin{array}{l}-57^{a} \\
-10^{b}\end{array}$ & $\begin{array}{r}+167^{a} \\
+61^{b}\end{array}$ & $\begin{array}{l}+35^{a} \\
+21^{b}\end{array}$ & $\begin{array}{r}+367^{a} \\
-47^{b}\end{array}$ & $\begin{array}{r}+154^{a} \\
+57^{b}\end{array}$ & $\begin{array}{r}+208^{a} \\
+65^{b}\end{array}$ \\
\hline
\end{tabular}

* Les chiffres suivis de la même lettre (dans les colonnes) ne sont pas significativement différents $(\mathrm{P}<0,05)$

$\mathrm{Sp}=$ saison des pluies $; \mathrm{Ss}=$ saison sèche

$\mathrm{Al}=$ argilo-limoneux $; \mathrm{Sa}=$ sablo-argileux 
pâturages, car elle prend aussi en compte la composition botanique, leur contribution spécifique et l'appétence de ces espèces.

Fréquence des feux, temps de repos après feux, rotations et charges : comparaison des systèmes 1 et 2 sur sol argilo-limoneux

Dans une deuxième série d'essais avec des animaux, la productivité du système 1 a été comparée à celle du système 2 (figure 4 ; tableau VIII).

Trois chargements (fort, modéré, faible), soit respectivement 2, 4 et 6 ha par animal ont été comparés pour le système 1, tandis que la charge du système 2 a été de 4 ha par animal.

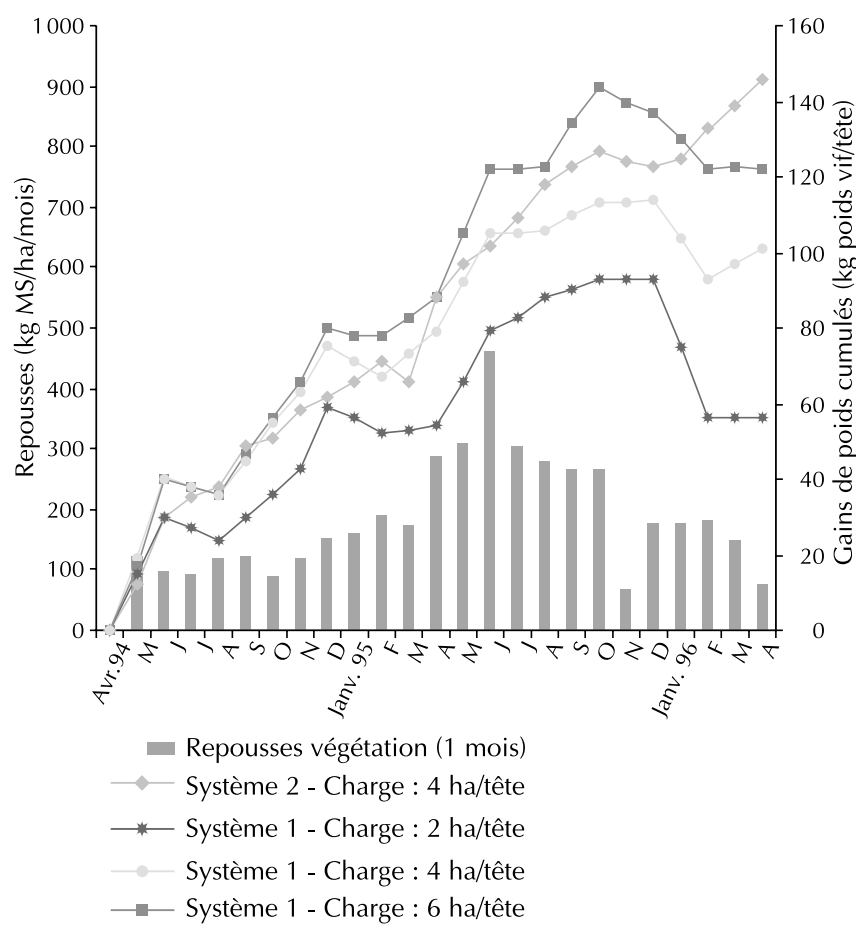

Figure 4 : comparaison de deux systèmes de gestion améliorée par les feux de prairies naturelles des Llanos sur sols argilo-limoneux selon les deux systèmes de feux. Gains de poids cumulés de jeunes bovins et repousses de quatre semaines.
Les courbes de la figure 4 montrent qu'il y a eu en général une bonne relation entre la production de repousses de quatre semaines et les gains de poids des animaux au cours des deux années d'observation.

Ainsi, durant la première Sp (d'avril à décembre 1994), en juin, juillet et août, la productivité du tapis herbacé a été relativement faible ; durant ces trois mois, une diminution de poids des animaux a été observée. La faible production du tapis herbacé semble liée aux très importantes précipitations durant cette période (très supérieures à la normale) qui a provoqué des inondations dans les prairies.

En Ss (de décembre 1994 à avril 1995), un ralentissement du gain de poids, voire des pertes de poids, ont été observés chez les animaux. Durant cette saison, il n'y a eu que quelques pluies mais les plantes ont pu profiter des réserves d'eau accumulées dans le sol durant la saison des pluies très pluvieuse. Durant cette saison, la bonne repousse du pâturage n'a pas profité aux animaux.

D'autres facteurs sont intervenus, comme l'insuffisance de Ms ingérée, la chaleur, etc. Une bonne corrélation entre gains de poids et productivité du pâturage a cependant existé durant la période de début des pluies (d'avril à juin 1995) : la croissance rapide de l'herbe a permis des gains de poids élevés. Elle correspond aussi à la période bien connue de compensation de poids après la $\mathrm{Ss}$.

Le tableau VIII permet de faire la comparaison et l'analyse détaillée (voir ci-après) des gains de poids des animaux durant les différentes saisons des deux années de l'expérimentation.

Au cours de la première $S p$ (d'avril à décembre 1994), avec une charge faible (6 ha par tête) ou moyenne (4 ha par tête), les gmq ont été significativement supérieurs dans le système 1. Dans le système 2 avec une charge forte, les gmq ont été comparables aux fortes charges ( 2 ha/tête/j) du système 1 .

Au cours de la Ss suivante (de décembre 1994 à mars 1995), les gmq ont été négatifs pour le système 1 avec une forte charge (- $111 \mathrm{~g} /$ tête/j), alors qu'ils sont restés légèrement positifs pour les autres charges dans ce système et ont été relativement élevés $(+289 \mathrm{~g})$ dans le système 2 , malgré un léger décrochement de la courbe en février.

Au terme de cette première année, dans le système 1 (rotation des feux sur quatre parcelles) avec des charges fortes ( 2 ha/tête), les

\section{Tableau VIII}

Comparaison des gains moyens quotidiens de bovins, durant les différentes périodes de l'année, dans différents modèles de gestion des pâturages naturels des Llanos de Colombie sur sols argilo-limoneux (en g)

\begin{tabular}{|c|c|c|c|c|c|c|c|}
\hline & $\begin{array}{c}\text { Sp } \\
\text { (avr.-déc. 94) }\end{array}$ & $\begin{array}{c}\text { Ss } \\
\text { (déc. 94-avr. 95) }\end{array}$ & $\begin{array}{l}\text { Sp + Ss } \\
(94-95)\end{array}$ & $\begin{array}{c}\text { Sp } \\
\text { (avr.-déc. 95) }\end{array}$ & $\begin{array}{c}\text { Ss } \\
\text { (déc. 95-avr. 96) }\end{array}$ & $\begin{array}{l}\text { Sp + Ss } \\
(95-96)\end{array}$ & Total essai \\
\hline \multicolumn{8}{|l|}{$\begin{array}{l}\text { Système } 1 \\
\text { (feu/4 mois) }\end{array}$} \\
\hline \multicolumn{8}{|l|}{ Charge } \\
\hline 2 ha/tête & $+234^{b^{*}}$ & $-111^{d}$ & $+147^{c}$ & $+164^{b}$ & $-289^{d}$ & $+5^{d}$ & $+76^{d}$ \\
\hline 4 ha/tête & $+298^{a}$ & $+34^{c}$ & $+215^{b}$ & $+147^{b}$ & $-102^{b}$ & $+60^{c}$ & $+138^{c}$ \\
\hline 6 ha/tête & $+317^{a}$ & $+69^{b}$ & $+210^{b}$ & $+210^{a}$ & $-133^{c}$ & $+90^{b}$ & $+165^{b}$ \\
\hline \multicolumn{8}{|l|}{$\begin{array}{l}\text { Système } 2 \\
\text { (feu mensuel } \\
\text { + rotation) }\end{array}$} \\
\hline Charge : 4 ha/tête & $+224^{b}$ & $+289^{a}$ & $+278^{a}$ & $+135^{c}$ & $+211^{a}$ & $+158^{a}$ & $+199^{a}$ \\
\hline
\end{tabular}

* Les chiffres suivis de la même lettre (dans les colonnes) ne sont pas significativement différents $(\mathrm{P}<0,05)$

$\mathrm{Sp}=$ saison des pluies $; \mathrm{Ss}=$ saison sèche 
gmq ont été très inférieurs à tous les autres modèles. Dans le système 1 , une charge supérieure à 4 ha par animal n'est donc pas envisageable en Ss sans complémentation.

Au cours de la seconde année (1995-96), les gmq ont été, dans les mêmes systèmes et en toute saison, inférieurs à ceux de l'année précédente. En $\mathrm{Sp}$, le système le moins performant a été le système $2(\mathrm{gmq}=+135 \mathrm{~g} / \mathrm{tête} / \mathrm{j})$, alors que dans le système 1 , les gmq ont été significativement supérieurs (respectivement $+164,+147$ et $+210 \mathrm{~g}$ pour les chargements de 2, 4 et 6 ha/tête).

Le système 1, avec faible charge, s'est donc montré le plus performant en $\mathrm{Sp}$, malgré une perte de poids des animaux constatée à partir de septembre 1995.

Au cours de la Ss de cette seconde année (de décembre 1995 à avril 1996), les gmq ont été négatifs dans le système 1, quelle qu'ait été la charge : - $289 \mathrm{~g}$ (!) pour les charges fortes, - $102 \mathrm{~g}$ pour les charges moyennes et - $133 \mathrm{~g}$ pour les charges faibles. Dans le système 2, au contraire, avec mise à disposition de jeunes repousses d'un mois, des gmq positifs de $211 \mathrm{~g}$ ont été observés.

Pour l'ensemble de la seconde année, le système 2, avec une charge moyenne, s'est montré à nouveau significativement supérieur au système 1, quelle qu'ait été la charge de ce dernier.

Finalement, pour l'ensemble des deux années, dans le système 2, les gmq ont été significativement supérieurs à ceux observés pour toutes les variantes de charge appliquées dans le système 1 et les gains de poids des animaux dans ce dernier ont pu être considérés comme inversement proportionnels à la charge appliquée au pâturage.

Pour les deux années, dans le système 2, des gains de poids importants en Ss ont été observés, alors qu'en Sp ceux-ci ont curieusement été inférieurs à toutes les variantes du système 1 .

Le comportement des animaux observé au pâturage pendant plusieurs mois par les auteurs $(1,4)$ et dont quelques résultats ont été résumés dans le tableau IX ont permis de formuler une explication.

Dans le système 2, en Sp, les animaux ont exploité, au cours des dix premiers jours de la rotation, pendant la majeure partie de la journée, les deux parcelles du même bloc où se trouvaient les plus jeunes repousses ( 29 p. 100 de la journée la parcelle la plus récemment brûlée, soit de 30 à 40 jours, et 55 p. 100 de la journée l'avant-dernière parcelle brûlée dans le bloc, dont les repousses avaient donc 90 à 100 jours), jusqu'à l'épuisement total de ces repousses et sans utiliser les repousses des autres parcelles du même bloc. Durant une seconde période, d'environ dix jours, les animaux ont exploité surtout les autres parcelles du bloc en se confinant, pendant 37 p. 100 de la journée sur les repousses de 20 et 28 semaines et pendant 26 p. 100 de la journée sur les repousses de 36 semaines et plus. Durant les dix derniers jours du passage sur un même bloc, les animaux ont exploité sans distinction d'âge toutes les parcelles.

Dans le système 1 (figure 1), les animaux ont exploité rapidement, pendant un peu plus d'un mois, la parcelle la plus récemment brûlée (dès le stade d'âge 0 ), puis ils se sont dispersés sur toute la superficie du parcours, exploitant aussi bien les repousses vertes les plus anciennes que les plus jeunes.

En conséquence, en $\mathrm{Sp}$ dans le système 1, les animaux ont consommé plus de matière sèche que dans le système 2. En effet, dans ce dernier, les animaux n'ont eu à leur disposition en permanence que 50 p. 100 de la superficie totale, alors que dans le système 1, les animaux ont eu 100 p. 100 du pâturage à leur disposition.

En Ss, dans le système 1, les animaux se sont donc eux-mêmes confinés sur la parcelle brûlée en décembre (= sur seulement 25 p. 100 du parcours), surpâturant les espèces appétées et ralentissant ainsi leur croissance par une limitation importante de leur indice de surface foliaire. Dans le système 2, les animaux ont parcouru les parcelles les plus récemment brûlées (surtout de 4 et 12 semaines de repousses) et ont eu à leur disposition durant ces quatre à cinq mois près de 50 p. 100 de la superficie totale $(8$ à 10 parcelles) puisqu'en saison sèche deux parcelles du même bloc ont été brûlées simultanément chaque mois.

Le comportement des animaux au pâturage et les superficies disponibles ont permis d'expliquer les gains de poids de ces deux systèmes : en saison des pluies, dans le système 1 , les animaux n'ont pas été limités par la surface à exploiter, alors qu'en Ss, les animaux de ce système 1 se sont limités à la surface récemment brûlée ( 25 p. 100 de la surface vite épuisée) ; dans le système 2, les animaux ont eu à leur disposition 50 p. 100 de la superficie totale (avec des jeunes repousses de bonne qualité d'un mois, renouvelables tous les mois).

\section{CONCLUSION}

Les différences de productivité des animaux exploitant les pâturages naturels de différents types de sols sont provenues surtout des différences de la valeur pastorale et, dans une moindre mesure, des différences de la valeur alimentaire de la biomasse moyenne. Il est nécessaire, pour bien mesurer la valeur d'un pâturage naturel, de connaitre non seulement sa productivité et sa valeur alimentaire globale, mais également la composition botanique du pâturage, la productivité de chaque espèce, leur valeur alimentaire et leur appétence qui permet alors de connaître la $\mathrm{Vp}$ du pâturage.

La comparaison de différents systèmes de gestion des pâturages naturels, qui utilisent le feu comme outil de gestion, a montré qu'en Ss, les brûlis en rotation tous les mois d'une petite partie du parcours (soit une mise à disposition des animaux chaque mois d'environ 10 p. 100 de la superficie totale du pâturage ayant des repousses d'un à deux mois d'âge) permet des gains de poids, alors

Tableau IX

Comportement sur le système 2 : pourcentage de consommation des repousses de différents âges

\begin{tabular}{lcccc}
$\begin{array}{l}\text { Age des repousses } \\
\text { (semaines) }\end{array}$ & $\begin{array}{c}\text { Saison sèche } \\
\text { (\%) }\end{array}$ & $\begin{array}{c}\text { Saison des pluies } \\
\text { Début du mois } \\
\text { de rotation (\%) }\end{array}$ & $\begin{array}{c}\text { Milieu du mois } \\
\text { de rotation (\%) }\end{array}$ & $\begin{array}{c}\text { Fin du mois } \\
\text { de rotation (\%) }\end{array}$ \\
\hline $4-8$ & 55 & 29 & 19 & 30 \\
$12-16$ & 32 & 55 & 18 & 12 \\
$20-32$ & 7 & 11 & 37 & 29 \\
$\geq 36$ & 6 & 5 & 26 & 29
\end{tabular}


qu'un unique feu en début de Ss sur seulement un quart du parcours ne le permet pas.

Le brûlage de la saison des pluies, chaque mois, d'une petite partie du parcours (5 p. 100) n'est pas souhaitable car les animaux se confinent sur les parcelles les plus récemment brûlées et n'utilisent pas les repousses vertes des parcelles un peu plus anciennes. Un brûlage de nettoyage et de régénération tous les quatre mois durant la saison des pluies, sur 25 p. 100 de la surface totale, paraît suffisant pour éliminer éventuellement les refus.

La rotation, entre deux blocs, qui permet d'exploiter des repousses de quatre semaines de bonne qualité, est hautement souhaitable car elle empêche la dégradation du pâturage. En effet, sur une parcelle non mise en défens un certain temps après feu, les animaux exploitent les espèces les plus appétées, en continu, à ras du sol, jusqu'à l'arrachement des touffes. Les espèces appétées qui persistent n'ont plus, d'autre part, la possibilité de produire des repousses et des réserves souterraines étant donné la forte diminution de leur indice de surface foliaire ; elles s'épuisent et disparaissent rapidement pour laisser la place à du sol nu ou à des espèces annuelles ou vivaces moins appétées ou de production inférieure (16).

Dans un ranch, il n'est pas nécessaire de dresser une clôture entre les deux blocs si ceux-ci sont suffisamment éloignés l'un de l'autre. En revanche, dans les Llanos, des pare-feu ou des coupe-feu d'au moins $2 \mathrm{~m}$ de large (pas plus, car la végétation ne dépasse que rarement $40 \mathrm{~cm}$ de hauteur moyenne et la biomasse $3 \mathrm{t} \mathrm{M} / \mathrm{ha}$ ) sont indispensables pour contrôler les brûlis dans les parcelles, surtout en Ss.

Cependant, ces améliorations de gains pondéraux sur pâturages naturels en saison sèche et même en saison des pluies ont aussi leur limite. Dans les hautes plaines planes des Llanos, là où des cultures fourragères sont possibles, ou dans les zones à relief plus marqué où des bas-fonds peuvent être aménagés et cultivés (serranilla), ces modèles de gestion doivent être intégrés, lorsque le contexte socio-économique le permet, à des systèmes d'élevage associant pâturages naturels, cultures fourragères (si possible association graminées-légumineuses) pour des réserves sur pied de saison sèche (banques de fourrage). Ceci permet, surtout en Ss, des gmq de plus de $330 \mathrm{~g}$, comme cela a été obtenu régulièrement en station $(10,15)$, ou des fourrages conservés (foins) et des complémentations avec des sous-produits agricoles ou agro-industriels, hélas encore assez rares dans cette région.

\section{Remerciements}

Nous remercions vivement H. Guerin du Cirad-emvt pour sa collaboration, la relecture du texte et ses nombreuses suggestions.

\section{BIBLIO GRAPHIE}

1. ALLARD G., 1995. Etude du comportement alimentaire des bovins et capacité d'exploitation de la savane colombienne. Rapport de stage de Dess, U niversité Paris XII Val de Marne, France, $85 \mathrm{p}$.

2. CESAR J., 1992. La production biologique des savanes de Côte d'Ivoire et son utilisation par I'homme : biomasse, valeur pastorale et production fourragère. Maisons-Alfort, France, Cirad-lemvt, $671 \mathrm{p}$.

3. COCHRANE T.T., SANCHEZ L.G., PORRAS J.A., DE AZEVEZO L.G., GARVER C.L., 1985. La tierra americana tropical. Cali, Colombia, CIAT/Brazilia, Brazil, EMBRAPA, $444 \mathrm{p}$.

4. CORBIN J., 1996. Etude d'un système d'exploitation de la savane des Llanos orientales de Colombie par rotation des brûlis. Mémoire de fin d'études, Istom, Cergy-Pontoise, France, 55 p. + annexes

5. DAGET P., GODRON M. (Coord.), 1995. Pastoralisme. Troupeaux espaces et sociétés. Paris, France, Aupel-U ref, $510 \mathrm{p}$.

6. DAGET P., POISSO NET J., 1971. Méthode d'analyse de la végétation des pâturages. Critère d'application. Ann. Agron., 22 : 5-41.

7. FISHER M.J., LASCANO C.E., VERA R.R., RIPPSTEIN G., 1992 Integrating the native savanna resource with improved pastures, pastures for the tropical lowlands: CIAT's Contribution. Cali, Colombia, CIAT, 228 p. (Publication N o. 211)

8. HU M PHREYS L.R., 1991. Tropical pasture utilisation. Cambridge, UK Cambridge University Press, $206 \mathrm{p}$

9. IEMVT-CIRAD/MINISTERE DE LA COOPERATION ET DU DEVELO PPEM ENT, 1990. Les feux de brousse. Maisons-Alfort, France, lemvt, 12 p. (Fiches techniques d'élevage tropical $n^{\circ} 3$ )

10. LASCANO C., 1991. Managing the grazing resource for animal production in savannas of the tropical America. Trop. Grassl., 25: 66-72.

11. PALADINES O., LEAL J., 1979. Pasture management and productivity in the Llanos O rientales of Colombia. In: Sanchez P.A. Tergas L.E. Eds, Pasture production in acid soils of the tropics. Cali,
Colombia, CIAT, p. 311-325.

12. RIPPSTEIN G., 1985. Etude sur la végétation de l'Adamaoua. Evolution, conservation, régénération et amélioration d'un écosystème pâturé au Cameroun. Maisons-Alfort, France, lemvt, 367 p. (Etudes et synthèses $n^{\circ}{ }^{14}$ )

13. RIPPSTEIN G., 1989. Une méthode d'étude et de classification des pâturages tropicaux. In : XVIe Congrès international des herbages, Nice, France, octobre 1989 , p. 1435-1436.

14. RIPPSTEIN G., 1991. Annual report; Tropical pasture program 19871991. Cali, Colombia, CIAT, 58 p.

15. RIPPSTEIN G., LASCANO C., DECAENS T., 1996. La production fourragère dans les savanes d'Amérique du Sud intertropicale. Fourrages, 145 : 33-52.

16. RIPPSTEIN G., SERNA R.A., ESCOBAR G., 1996. Dinámica de la vegetación de la sabana nativa sometida a quema, pastoreo y diferentes formas de manejo en los Llanos orientales de Colombia. Taller regional. agrociencia y tecnología. Siglo XXI, O rinoquia Colombiana. Meta, Colombia, noviembre 13-15 de 1996, 12 p. + fig.

17. SALINAS J.G., GARCIA R., 1985. Métodos quimicos para el análisis de suelos acidos y plantas forrajeras. Cali, Colombia, CIAT, 83 p.

18. SARMIENTO G., 1990. Ecología comparada de ecosistemas de sabanas en América del Sur. In : Sarmiento G. ed. Las sabanas americanas. Aspecto de su biogeografía, ecología y utilización. Acto del Symposio IUBS,MAB-UNESCO. Guanare, Venezuela. Centro de Investigaciones Ecológicas de los Andes Tropicales. Mérida, Venezuela, Facultad de Ciencias, U niversidad de los Andes, p. 15-56.

19. TILLEY J.M.A., TERRY R.A., 1963. A two-stage technique for the in vitro disgestion of forage crops. J. Br. Grassl. Soc., 18: 104-111. 


\section{Summary}

Rippstein G., Allard G., Corbin J. Fire M anagement of N atural G rasslands and Cattle Productivity in the Lower Eastern Plains of Colombia

The productivity and nutritive value of various natural grasslands of the lower eastern plains (Llanos) of Colombia, fire-managed annually during the dry season, were usually low. Annual biomass was 2-3.5 tons of dry matter (DM) per hectare and total crude protein content of fodder was $5-11 \%$ of DM depending on the season and phenological stage. Under traditional managing conditions (non-stop grazing, no fire or fire at the beginning of the dry season, stocking rate of 8-10 ha per animal), cattle weight gains were low during the rainy season (average daily weight gains between $150-300 \mathrm{~g})$ and cattle usually lost weight during the dry season (150-250 g per day). During in-station experiments, significant differences in animal productivity were observed based on the botanical content and, even more so, on the pastoral value (a grassland value scale that combined productivity, nutritive value and desire of the animal for the species) of the natural grassland, which were determined by the soil texture (either sandy-clay or clay-silty). Regrowth occurred as a result of annual fires in the dry season. $\mathrm{N}$ evertheless, the year after year repetition of these fires associated with free non-stop grazing negatively modified the flora. The authors tested two grassland-burning sequential systems in a set of parcels used in rotation. The systems allowed burning intervals of 16 months and provided the cattle with regrowths protected from grazing every four weeks. Even with an average annual stocking rate multiplied by two (4 ha per head), the individual weight gains of growing cattle were significantly improved without degrading the vegetation.

Key words: Cattle - G razing system - Grassland management Stocking density - Rotational grazing - Regrowth - Nutritive value - Plain - Colombia.

\section{Resumen}

Rippstein G ., Allard G., Corbin J. Gestión mediante fuegos de los pastizales naturales y la productividad de los bovinos en las praderas de las Ilanuras bajas orientales de Colombia

La productividad y el valor alimenticio de los diferentes tipos de pastizales naturales de las Ilanuras bajas orientales de Colombia (Llanos), manejadas mediante fuegos anuales durante la estación seca, es generalmente bajo: las biomasas aéreas anuales fueron del orden de 2 a 3,5 toneladas de materia seca por hectárea y los contenidos en materias nitrogenadas totales de los pastos estuvieron comprendidos entre 5 y $11 \%$ de la materia seca según las estaciones y el estado fenológico. Bajo condiciones tradicionales de explotación (pastoreo continuo, sin fuego o con fuegos en el inicio de la estación seca, carga de 8-10 ha por animal), las ganancias de peso de los bovinos son bajas durante la estación de las lluvias (ganancias cotidianas medias de menos de 150 a $300 \mathrm{~g}$ ) y durante la estación seca se dan generalmente pérdidas de peso (150 a 250 g por día). Experimentos llevados a cabo en la estación mostraron una diferencia significativa de la productividad de los animales según la composición botánica 0 , aun mejor, el valor pastoril (índice sintético del valor del pasto integrando productividad, valor nutritivo y palatabilidad de las especies) de los pastos naturales, según se encuentren en suelos areno-arcillosos o arcillo-limosos. Los fuegos anuales durante la estación seca permitieron la producción de retoños verdes pero, repetidos año con año y asociados con el pastoreo libre continuo, modificaron negativamente la flora. Sobre un conjunto de parcelas en rotación, los autores prueban dos sistemas en secuencia de fuegos, permitiendo espaciarlos de 16 meses y poner a la disposición del ganado los retoños protegidos del pastoreo cada cuatro semanas. A pesar de una duplicación de la carga media anual (4 ha por cabeza), las ganancias de peso individuales de los bovinos en crecimiento mejoraron significativamente y este resultado se obtuvo sin degradación de la vegetación.

Palabras clave: Ganado bovino - Sistema de pastoreo Manejo de praderas - Carga ganadera - Pastoreo rotacional Rebrote - Valor nutritivo - Llanura - Colombia. 